\title{
Biophysics
}

\section{Crystallin measurements in the intact eye lens}

\section{from Christine Slingsby}

A FINE illustration of the more gentle ways of physicists than of biochemists, who have traditionally ripped cells apart to investigate - with great success - their macromolecular organization, is contained in a recent issue of Proceedings of the National Academy of Sciences U.S.A. In it, Sun et al. report the use of a light-scattering technique to measure the accumulation of $\delta$-crystallin in the intact eye lens of chickens'.

By focusing a small beam of laser light onto discrete regions of an intact lens and analysing the correlation time of the scattered light intensity fluctuations, Sun et $a l$. were able to observe brownian motions within the lens. Because the dimensions of the lens cell are large compared with the wavelength of light, the observed brownian motions are of cytoplasmic constituents of the cells. The motions depend on both the size of the scattering elements and their interactions with other molecules. The observed correlation function is therefore a composite of all the random walks of cytoplasmic constituents over distances comparable to the light wavelength but it is possible, in principle, to sort out molecular details of individual components if they are homogeneously dispersed in a single phase.

The diffusion coefficient of a macromolecule can be determined from the relaxation time of the scattered light intensity fluctuations, which is related to the correlation length by the Stokes-EinsteinKawasaki-Ferrel formula. When interactions between macromolecules are negligible the correlation length is equal to the hydrodynamic radius. When the correlation lengths of components in cells deviate moderately from those measured in homogeneous solutions, it is because of that ephemeral property of matter - shortrange order. On the other hand, if a solution or gel is near a critical point, so that different phases coexist yet are clearly distinguishable, the interactions become long range, the measured correlation length is very large and the solution becomes turbid ${ }^{2,3}$. The correlation length of $\delta$-crystallin measured within the lens by Sun et al. was only $20 \AA$ longer than the hydrodynamic radius measured from a monodisperse solution, in line with evidence that only short-range order is necessary for the transparency of the lens ${ }^{4,5}$.

The fewer the components within a cell, the more reliable the method of determining quantitative parameters of individual macromolecules. Embryonic chicken lenses are well suited in this respect because the cytoplasm is composed largely of cyto- skeletal filaments ${ }^{6}$ and $\delta$-crystallin, a tetramer of molecular weight near 200,000 (ref.7). In keeping with this description, Sun et al. find two correlation lengths, one very large $(6,700 \AA)$ and one small $(65 \AA)$, corresponding to these components. From the amplitude of the scattered light fluctuations in lenses from embryos and chicks of different ages, Sun et al. were able to determine the relative concentration of $\delta$ crystallin within lens cells during the course of the first forty days of development.

Interesting phenomena relating to lens biology discovered by other techniques could now be brought into the field of view of light-scattering science. Lens differen-

tiation requires growth factors from the retina $^{7}$; alterations in the ionic environment of lens cells regulate the size of $\delta$ crystallin subunit to be synthesized ${ }^{8}$; and calcium levels affect the association in vitro of $\delta$-crystallin to membranes. These and other physiological responses could now be non-invasively investigated in intact cells. Another obvious target for investigation would be the influence of the cytoskeleton of the lens cell on the mobility of its cytoplasmic constituents.

1. Sun, S-T., Tanaka, T., Nishio, I., Peetermans, J., Maizel, J.V. \& Piatigorsky, J. Proc, natn. Acad. Sci. U.S. A. 785 (1984).

2. Benedek, G.B. Polarization, Matter and Radiation (Presses Universitaire de France, Paris, 1969)

Tanaka, T. Scient. Am. 244, 110 (1981)

4. Benedek, G.B. Appl. Optics 10, 459 (1971)

5. Delaye, M. \& Tardieu, A. Nature 302, 415 (1983).

6. Maisel, H. et al. Molecular and Cellular Biology of the Eye Lens (ed. Bloemendal, H.) 49 (Wiley, New York, 1981).

Piatigorsky, J. Differentiation 19, 134 (1981)

8. Shinohara, T. \& Piatigorsky, J. Nature 270, 406 (1977).

Christine Slingsby is in the Department of Crystallography, Birkbeck College, University of London, Malet Street, London WCI 7 HX.

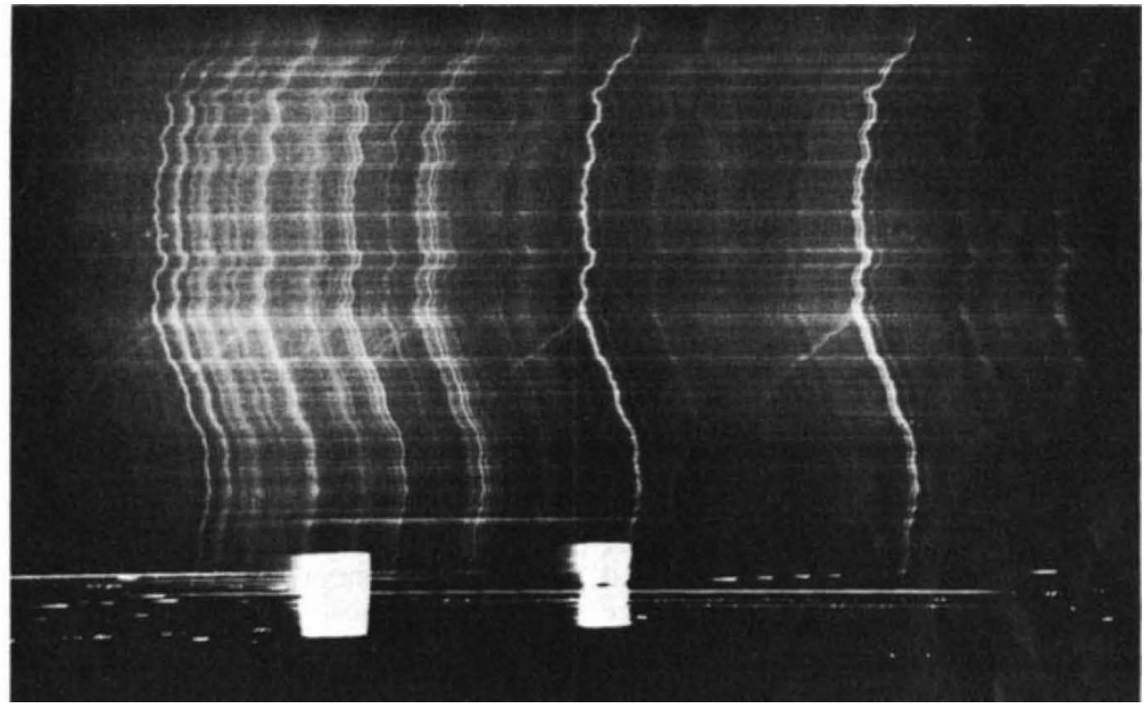

ALTHOUGH serious scientific work on the spectra of lightning dates from early this century, with the direct time-resolved photography of Sir Charles Boys and the slitless spectroscopy of E. C. Pickering, the manufacture of diffraction gratings with a large surface area of high accuracy and uniformity produced a revolution in lightning spectroscopy. In 1960, applying a Bausch \& Lomb replica transmission grating to an f/8 Aero-Tessar camera, Leon Salanave (then with the Institute of Atmospheric Physics at the University of Arizona) captured this historic stroke of lightning. The spectrum of a single stroke is seen dispersed at $25 \AA \mathrm{mm}^{-1}$ in first order, running from $380 \mathrm{~nm}$ at far left to $620 \mathrm{~nm}$ at extreme right.

The photograph may be viewed as a three-dimensional graph with position as the ordinate, wavelength as the abscissa and intensity varying from bright knots ('hot spots') to invisibility at any given point. The variation in intensity runs both vertically in space and horizontally in wavelength. All the brightest lines are attributable to $\mathrm{N}$ (II), but other prominent lines belong to $\mathrm{N}$ (I), $\mathrm{O}$ (I) and $\mathrm{O}$ (II). If the spectrum had run on a bit further to the right, the $\mathrm{H} \alpha$ line of hydrogen would have been conspicuous; the hydrogen originates in the dissociation of water vapour by the electric spark. Water vapour also makes its presence known explicitly towards the red end of the spectrum, where it is manifest as a broad dark band intersected by lines of N (II)

Writing of the historic significance of the photograph, Salanave comments, "The importance of these slitless spectra, dating from 1960 on, lies in the fact that from them (after time resolution into individual strokes) channel temperatures near peak luminosity could be reliably determined for the first time". Jon Darius One of the photographs in an exhibition Beyond Vision at the Science Museum, London from 19 April. A book with the same title will be published in April by Oxford University Press. Reproduced by courtesy of E. P. Krider, University of Arizona. 